bioRxiv preprint doi: https://doi.org/10.1101/2021.08.12.456140; this version posted August 13,2021 . The copyright holder for this preprint (which was not certified by peer review) is the author/funder, who has granted bioRxiv a license to display the preprint in perpetuity. It is made available under aCC-BY 4.0 International license.

Implicit adaptation of withheld movements - 1

\title{
Learning from the path not taken: Sensory prediction errors are sufficient for implicit adaptation of withheld movements
}

\author{
Olivia A. Kim¹, Alexander D. Forrence ${ }^{2}$, and Samuel D. McDougle ${ }^{2}$ \\ 1. Department of Psychology, Princeton University, Princeton, NJ 08544 \\ 2. Department of Psychology, Yale University, New Haven, CT 06511
}

Correspondence to:

Olivia Kim

oakim@princeton.edu

Number of Pages: 31

Number of Figures in Main Text: 3

Number of Supplementary Figures: 5

Number of Supplementary Tables: 3

References: 43

Number of Characters:

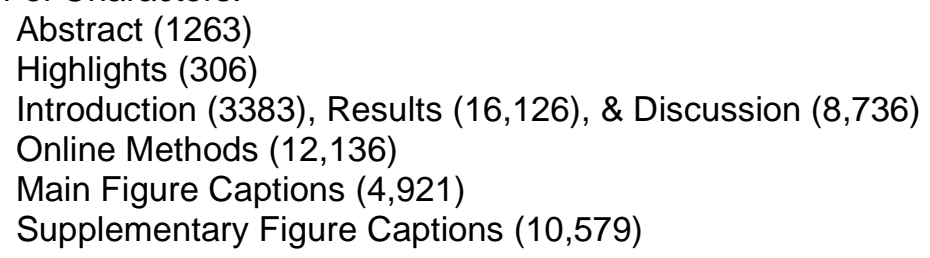

\section{AUTHOR CONTRIBUTIONS}

Conceptualization, OK, AF, \& SM; Methodology, OK, AF, \& SM; Software, OK \& AF; Validation, OK \& AF; Formal Analysis, OK \& AF; Investigation, OK \& AF; Resources, OK \& SM; Data Curation, OK \& AF; Supervision, SM; Project Administration, SM; Funding Acquisition, SM; Writing - original draft, OK; Writing - review \& editing, OK, AF, \& SM

\section{CONFLICT OF INTEREST}

The authors declare no competing financial interests. 
Implicit adaptation of withheld movements - 2

47

48

49

50

51

52

53

54

55

56

57

58

59

60

61

62

63

64

65

66

67

68 69

70

71

\section{ABSTRACT}

Current theories of motor control emphasize forward models as a critical component of the brain's motor execution and learning networks. These internal models are thought to predict the consequences of movement before sensory feedback from these movements can reach the brain, allowing for smooth, continuous online motor performance and for the computation of prediction errors that drive implicit motor learning. Taking this framework to its logical extreme, we tested the hypothesis that movements are not necessary for the generation of predictions, the computation of prediction errors, and implicit motor adaptation. Human participants were cued to move a computer mouse to a visually displayed target and were subsequently cued to withhold those movements on a subset of trials. Visual errors displayed on both trials with and without movements to the target induced single-trial learning. Furthermore, learning on trials without movements persisted when accompanying movement trials were never paired with errors and when movement and sensory feedback trajectories were decoupled. These data provide compelling evidence supporting an internal model framework in which forward models generate sensory predictions independent of the generation of movements.

\section{HIGHLIGHTS}

- The brain can learn to update movements that are not performed, representing a mechanism for learning based only on movement planning and sensory expectation.

- Supports a fundamental role for prediction in adaptation.

- Provides further support for the role of forward models in predictive motor control.

\section{KEYWORDS}

predictive coding, mental imagery, supervised learning, cerebellum 
Implicit adaptation of withheld movements - 3

\section{INTRODUCTION}

Forward models are thought to guide our motor behaviors by providing an internal representation of body-environment interactions. A key feature of these models is their ability to predict the consequences of movements before relevant sensory feedback can reach the motor controller. As delays between movements and sensory feedback processing are an inherent byproduct of the time needed for signal detection and neural propagation, forward models' predictive capacity is essential to motor control. ${ }^{1}$ When sensory feedback indicates that a prediction of a forward model is incorrect, the resulting sensory prediction error is used to update future predictions and optimize motor commands. ${ }^{2-5}$ Such motor adaptation proceeds implicitly regardless of task goals, reflecting the powerful influence of prediction errors on the motor system's internal models. ${ }^{6-8}$

The predictive capacity of forward models led us to a strong logical inference: the forward model should predict and learn from the sensory consequences of an intended movement regardless of whether that movement occurs. That is, because movements are synchronous with the sensory outcomes that they produce, motor execution cannot itself be necessary for the generation of predictions by the forward model or the ensuing computation of sensory prediction error. Indirect evidence for this claim comes from observations that the brain predicts and accounts for the sensory consequences of movement during motor imagery in the absence of overt motor behavior. ${ }^{9-11}$ This suggests that prediction alone should be sufficient for the computation of the sensory prediction errors that drive motor adaptation. An internal model for motor control should thus be effectively trained with only two constituent events - generation of a sensory prediction and observation of sensory feedback (Fig. 1a). Here, we asked if performing a particular movement is necessary for its adaptation.

To do so, we measured trial-by-trial implicit adaptation during a visuomotor task in which human participants saw visual feedback while performing - or withholding - hand movements, using a so-called "Go/No-Go" paradigm. At the onset of each trial, a "Go" cue was presented, instructing participants to make a movement through a visual target. On some trials, a "No-Go" cue was presented shortly after presenting the "Go" cue, instructing participants to withhold the movement (Fig. 1b). We reasoned that presenting the "Go" cue on "No-Go" trials would be a sufficient stimulus to induce prediction generation and support sensory prediction error computation, given that prior work has shown that cued - but ultimately withheld - 
Implicit adaptation of withheld movements - 4

100

101

102

103

104

105

106

107

108

a

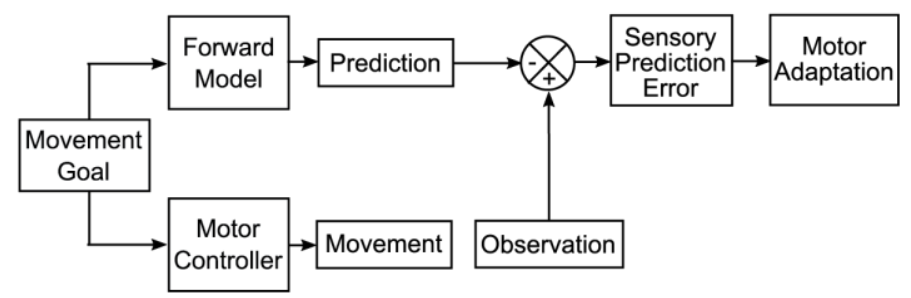

C are executed. Triplet Design

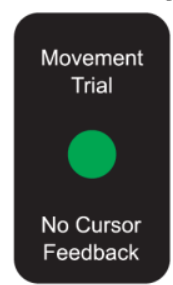

Trial 1
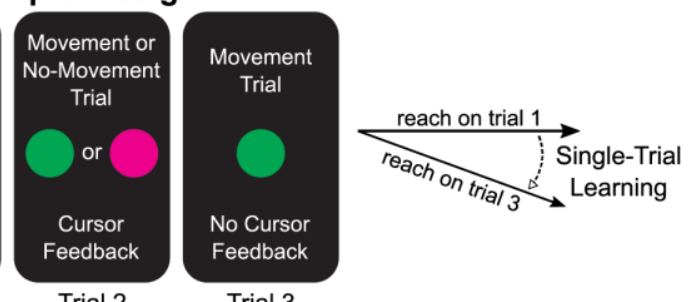

Trial 2

Trial 3 reach angles on the flanking trials.

movements can still serve as an input to the forward model and support the generation of differential sensory predictions. ${ }^{12}$ To isolate implicit adaptation, we employed a recently-developed approach that requires participants to aim their movements directly for presented targets and disregard visual feedback..$^{8,13-17}$ We predicted that single-trial motor adaptation would occur following both typical movement (Go) trials that generated sensory error, as well as trials where movements were withheld (No-Go) but "simulated" sensory errors were observed. If confirmed, this result would demonstrate that sensory prediction errors can cause adaptation of selected movements, regardless of whether those movements

b Movement Trials

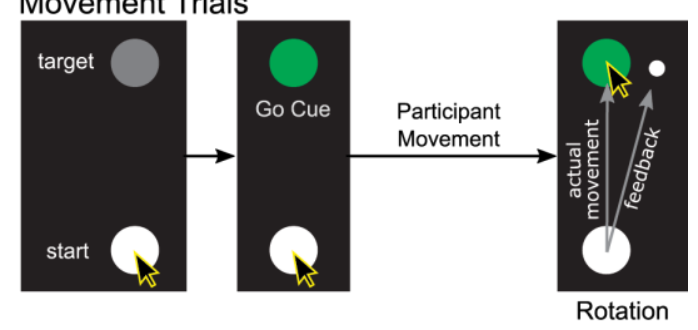

No-Movement Trials

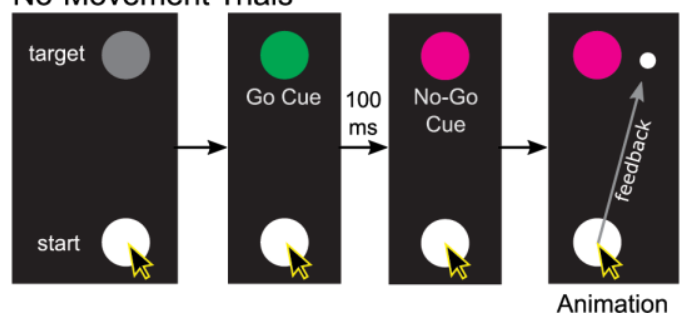

Figure 1. Schematics showing the proposed learning framework and task design. (a) Schematic showing how the forward model may support motor adaptation in the presence of sensory feedback not causally related to self-generated movement. (b) Schematic of events on trials with visual feedback. Participants brought their mouse cursor (black pointer) to the starting location to initiate a trial. On Movement trials (top), the target turned green ('Go Cue'), cueing participants to move the mouse through the target. Depending on the trial type, participants either observed a white feedback cursor move along their mouse trajectory, or along a rotated trajectory ('Rotation'). On No-Movement trials (bottom), the target turned magenta 100 ms after turning green, cueing participants to withhold movement on this trial. After a delay, an animation showing the feedback cursor moving straight to the target or $15^{\circ}$ off-target played ('Animation'). The black mouse pointer is shown in the figure for illustrative purposes but was not visible during the experiment. (c) Schematic showing the trials that constituted a triplet and how single-trial learning (STL) was computed. Triplets were composed of 2 Movement trials without visual feedback flanking either a Movement or a No-Movement trial with visual feedback. STL was measured as the difference between 
Implicit adaptation of withheld movements - 5

124

125

126

127

128

129

130

131

132

133

134

135

136

137

138

139

140

141

142

143

144

145

146

147

148

149

150

151

\section{RESULTS}

Simulated and genuine visuomotor rotations cause motor adaptation

We measured implicit motor adaptation in humans $(n=35)$ performing straight hand movements during a computerized version of a visuomotor adaptation task (Fig. 1b). Trials were organized into triplets, such that each trial with feedback was flanked by trials without feedback, allowing for a reliable measurement of single-trial learning (STL) as the difference between the third and first trial of each triplet (Fig. 1c). Trials in which feedback was presented were designated as either "Movement" trials (a "Go" signal prompted movement) or No-Movement trials (a "No-Go" signal prompted the withholding of movement). On Movement trials, feedback either involved a visuomotor error $\left( \pm 15^{\circ}\right.$ rotation added to the visual cursor path; + = clockwise) or was veridical (no rotation added). On No-Movement trials, sensory feedback involved a simulation of the cursor's path, using timing variables based on ongoing participant performance (Fig. 2a, see Methods). All flanking trials were "Go" trials and thus required movements. This straightforward design allowed us to test the hypothesis that forward-model-based motor adaptation does not require a causal link between movement and sensory feedback (Fig. 1a).

Consistent with our predictions, rotated cursor paths on Movement and No-Movement trials both induced significant adaptation: Movement trajectories on the following trial tended to shift opposite the direction of the cursor path. This was reflected by STL observed when participants experienced non-zero rotations of cursor feedback (Fig. 2b-c). A linear mixed model (LMM; fixed effects: rotation [15 counterclockwise $\{\mathrm{CCW}\}, 0^{\circ}$, and $15^{\circ}$ clockwise $\{\mathrm{CW}\}$ ], movement condition [Movement, No-Movement], rotation $\mathrm{x}$ movement condition interaction; random effects: participant) was fit to participants' triplet-wise STL performance. The LMM revealed significant main effects of rotated cursor feedback $(F(2,1959)=$ 131.13, $p=2.2 \times 10^{-16}$, partial $\left.R^{2}=0.12\right)$ and movement condition $\left(F(1,1982)=4.23, p=0.04\right.$, partial $R^{2}=$ $0.01)$, as well as a significant interaction $\left(F(2,1965)=9.71, p=6.36 \times 10^{-5}\right.$, partial $\left.R^{2}=0.002\right)$. Post-hoc pairwise comparisons of the estimated marginal means from the model (Supplementary Table 1) support the claim that rotated feedback induced a statistically significant degree of STL on both Movement $\left(0^{\circ}\right.$ vs $15^{\circ} \mathrm{CW}: t(1964)=8.94, p_{a d j}=3.88 \times 10^{-18}$, Cohen's $d=0.64 ; 0^{\circ}$ vs $15^{\circ} \mathrm{CCW}: t(1971)=7.143, p_{a d j}=2.89$ $\times 10^{-12}$, Cohen's $\left.d=0.51\right)$ and No-Movement trials $\left(0^{\circ}\right.$ vs $15^{\circ} \mathrm{CW}: t(1964)=4.24, p_{a d j}=3.49 \times 10^{-5}$, Cohen's $d=0.36 ; 0^{\circ}$ vs $15^{\circ} \mathrm{CCW}: t(1966)=3.44, p_{a d j}=0.0008$, Cohen's $\left.d=0.30\right)$. Adaptation in the presence of a 

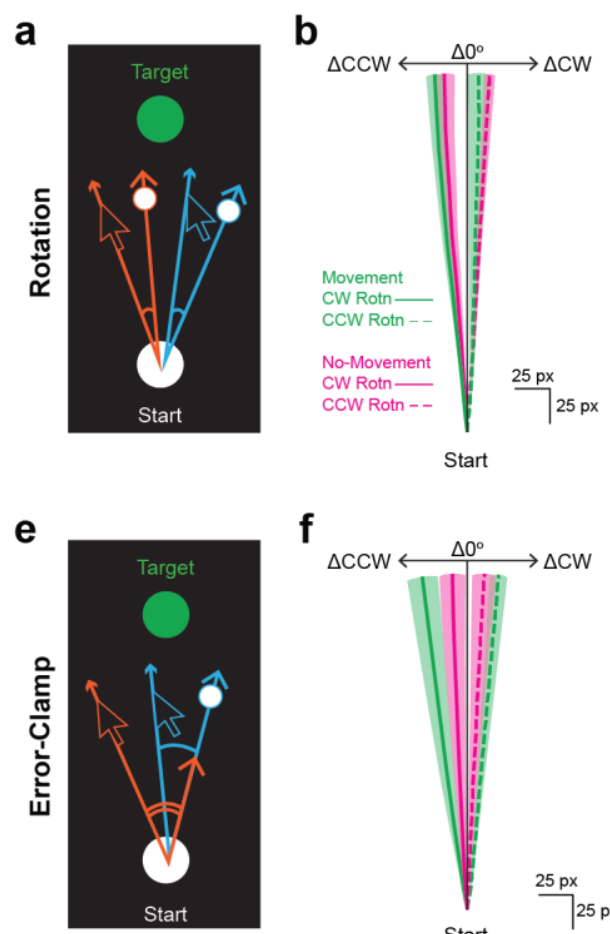

f

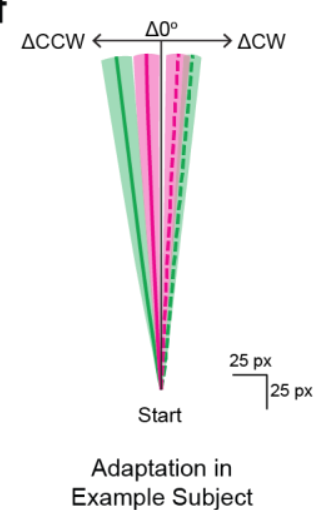

C

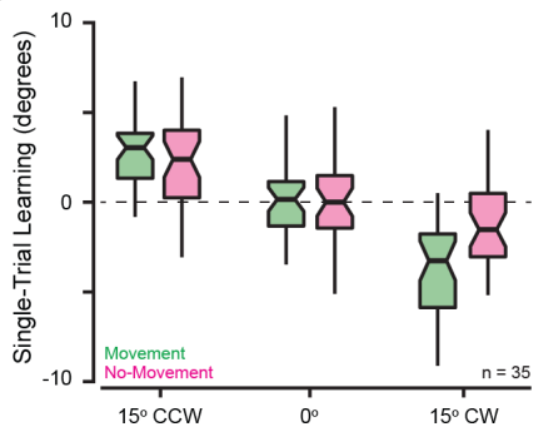

g

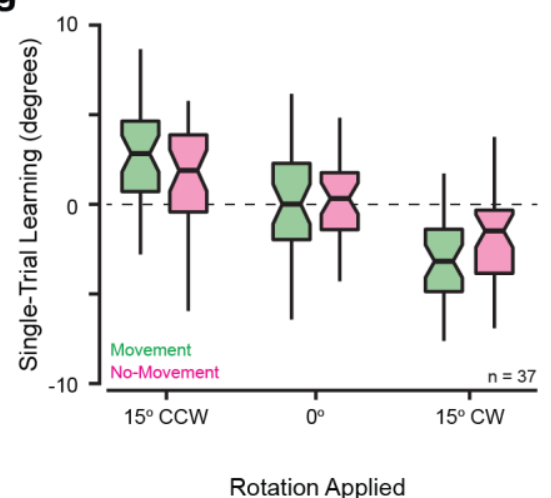

d

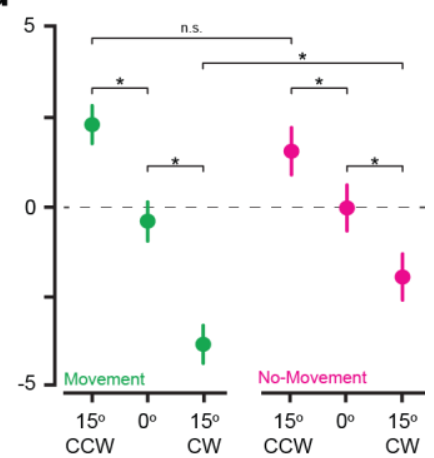

h

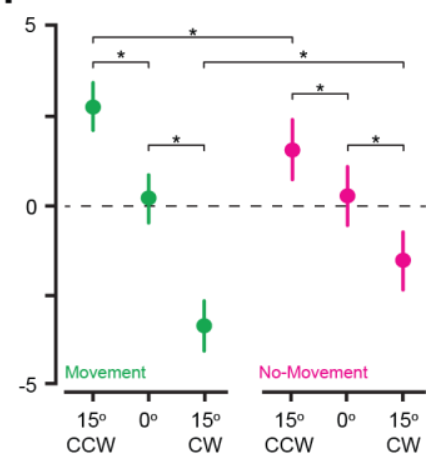

Rotation Applied
Figure 2. Single-trial learning in response to errors on Movement and No-Movement trials in the context of visuomotor rotations and error-clamps. (a) Schematic of the relationship between mouse movement and cursor feedback on Movement trials where a rotation was applied to visual feedback. Corresponding mouse (black pointer) and cursor (white circle) movement vectors are color coded. (b) An example participant's mean \pm SEM changes in reach paths across triplets with a rotation applied. Green shading indicates that data are from triplets in which a perturbation was applied on a Movement trial, while magenta shading indicates that the perturbation was applied on a No-Movement trial. Solid lines indicate that a clockwise (CW) rotation was applied on the relevant perturbation trial, while dashed lines indicate that a counterclockwise (CCW) rotation was applied. (c) Boxplot showing change in hand angle across Movement (green) and No-Movement (magenta) triplets in an experiment where feedback on Movement trials was rotated relative to mouse position. In the reference frame used in these experiments, positive changes in hand angle are CW. (d) Estimated marginal means $\pm 95 \%$ confidence intervals from the linear mixed models (LMM) fit to each participant's single-trial learning performance summarized in (c). Please refer to Supplementary Table 1 for details on all post-hoc comparisons. (e - h) As in (a - d), but for an experiment where cursor feedback on Movement trials was error-clamped relative to target location. Please refer to Supplementary Table 2 for details on post-hoc comparisons. Boxplot center: median, notch: 95\% confidence interval of the median, box edges: $1^{\text {st }}$ and $3^{\text {rd }}$ quartiles, whiskers: most extreme value within $1.5^{*}$ interquartile range of the median. Statistical significance $\left({ }^{*}=p<0.05\right.$; n.s. $\left.=p \geq 0.05\right)$ is indicated for selected comparisons. Abbreviations: $\Delta$ - change, CW - clockwise, CCW - counterclockwise. 


\section{Supplementary Table 1.}

Pairwise post-hoc comparisons between estimated marginal means in Fig. $2 d$

\begin{tabular}{|c|c|c|c|c|c|c|c|c|}
\hline \multicolumn{2}{|c|}{ Group 1} & \multicolumn{2}{|c|}{ Group 2} & \multirow{2}{*}{$\begin{array}{l}\text { Est. } \\
\text { Diff. }\end{array}$} & \multirow[t]{2}{*}{$\mathrm{t}$} & \multirow{2}{*}{ df } & \multirow{2}{*}{$\begin{array}{c}\text { FDR- } \\
\text { adjusted } p\end{array}$} & \multirow{2}{*}{$\begin{array}{c}\text { Cohen's } \\
\text { d }\end{array}$} \\
\hline $\begin{array}{l}\text { Trial } \\
\text { Type }\end{array}$ & Rotation & $\begin{array}{l}\text { Trial } \\
\text { Type }\end{array}$ & Rotation & & & & & \\
\hline M & $0^{\circ}$ & M & $15^{\circ} \mathrm{CW}$ & $3.77^{\circ}$ & 8.942 & 1964 & * $3.88 \times 10^{-18}$ & 0.65 \\
\hline M & $0^{\circ}$ & M & $15^{\circ} \mathrm{CCW}$ & $2.96^{\circ}$ & 7.14 & 1958 & ${ }^{*} 2.89 \times 10^{-12}$ & 0.51 \\
\hline M & $15^{\circ} \mathrm{CW}$ & M & $15^{\circ} \mathrm{CCW}$ & $6.73^{\circ}$ & 16.20 & 1960 & $* 1.45 \times 10^{-54}$ & 1.15 \\
\hline No-M & $0^{\circ}$ & No-M & $15^{\circ} \mathrm{CW}$ & $2.12^{\circ}$ & 4.24 & 1962 & $* 3.49 \times 10^{-5}$ & 0.36 \\
\hline No-M & $0^{\circ}$ & No-M & $15^{\circ} \mathrm{CCW}$ & $1.74^{\circ}$ & 3.44 & 1966 & * 0.0008 & 0.30 \\
\hline No-M & $15^{\circ} \mathrm{CW}$ & No-M & $15^{\circ} \mathrm{CCW}$ & $3.86^{\circ}$ & 7.61 & 1964 & $* 1.27 \times 10^{-13}$ & 0.66 \\
\hline M & $0^{\circ}$ & No-M & $0^{\circ}$ & $0.40^{\circ}$ & 0.88 & 1975 & 0.38 & -- \\
\hline M & $15^{\circ} \mathrm{CW}$ & No-M & $15^{\circ} \mathrm{CW}$ & $2.06^{\circ}$ & 4.44 & 1974 & $* 1.67 \times 10^{-5}$ & 0.35 \\
\hline$M$ & $15^{\circ} \mathrm{CCW}$ & No-M & $15^{\circ} \mathrm{CCW}$ & $0.82^{\circ}$ & 1.76 & 1971 & 0.09 & -- \\
\hline
\end{tabular}

Note. Abbreviations: M - Movement; No-M - No-Movement; Est. Diff. - Estimated Differences in degrees. Note that degrees of freedom pertain to the inputs to the LMM and are estimated using the Kenward-Rogers approach. Statistically significant $p$-values are indicated with asterisks. ${ }^{*} p<0.05$.

To address whether the STL measured represented genuine implicit learning, we checked whether adaptation persisted beyond the trial after an error was experienced. We examined participants' hand angles on the second trial after a perturbation relative to the pre-perturbation baseline trial (i.e., hand angle on trial 1 of triplet $t+1$ relative to hand angle on trial 1 of triplet $t$, henceforth referred to as remembered STL, Supplementary Fig. 1a). Because visual feedback was withheld on both trial types, this approach provides a relatively pure measure of persistent learning in the absence of error-driven changes in performance. Hand angle appeared to remain adapted in the direction opposite the rotation on trials with nonzero perturbations regardless of movement condition (Supplementary Fig. 1b-c), suggesting that genuine implicit learning was observed in response to errors under both movement conditions. Participantwise GLMs fit to individual subjects' remembered STL measures (rotation, movement condition, and their interaction included as predictors) support this interpretation: the distribution of estimated coefficients for 
bioRxiv preprint doi: https://doi.org/10.1101/2021.08.12.456140; this version posted August 13, 2021. The copyright holder for this preprint (which was not certified by peer review) is the author/funder, who has granted bioRxiv a license to display the preprint in perpetuity. It is made available under aCC-BY 4.0 International license.

Implicit adaptation of withheld movements -8

190

rotation as a predictor was significantly different from zero (Wilcoxon's signed-rank test: $V=25, p_{\text {adj }}=1.58$

$\left.191 \times 10^{-7}, r=-0.80\right)$, whereas estimated movement coefficients $\left(V=228, p_{a d j}=0.24\right)$ and movement/rotation

192 interaction coefficients $\left(V=365, p_{a d j}=0.42\right)$ did not differ significantly from 0 (Supplementary Fig. 1d).

193 Importantly, additional participant-wise GLMs fit to the adaptation observed the trial after error presentation

194 (i.e., the STL measurements shown in Fig. 2) with the perturbations applied on that and the preceding

195 triplet used as predictors showed that STL was not attributable to errors observed on preceding Movement

196 triplets (Wilcoxon's signed-rank test: $V=283, p_{a d j}=0.70$; Supplementary Fig. 2a). Thus, while STL

197 persisted at least one trial beyond the triplet in which an error was observed, STL observed on No-

198 Movement trials was not attributable to residual adaptation from preceding triplets with errors on Movement

199 trials. 

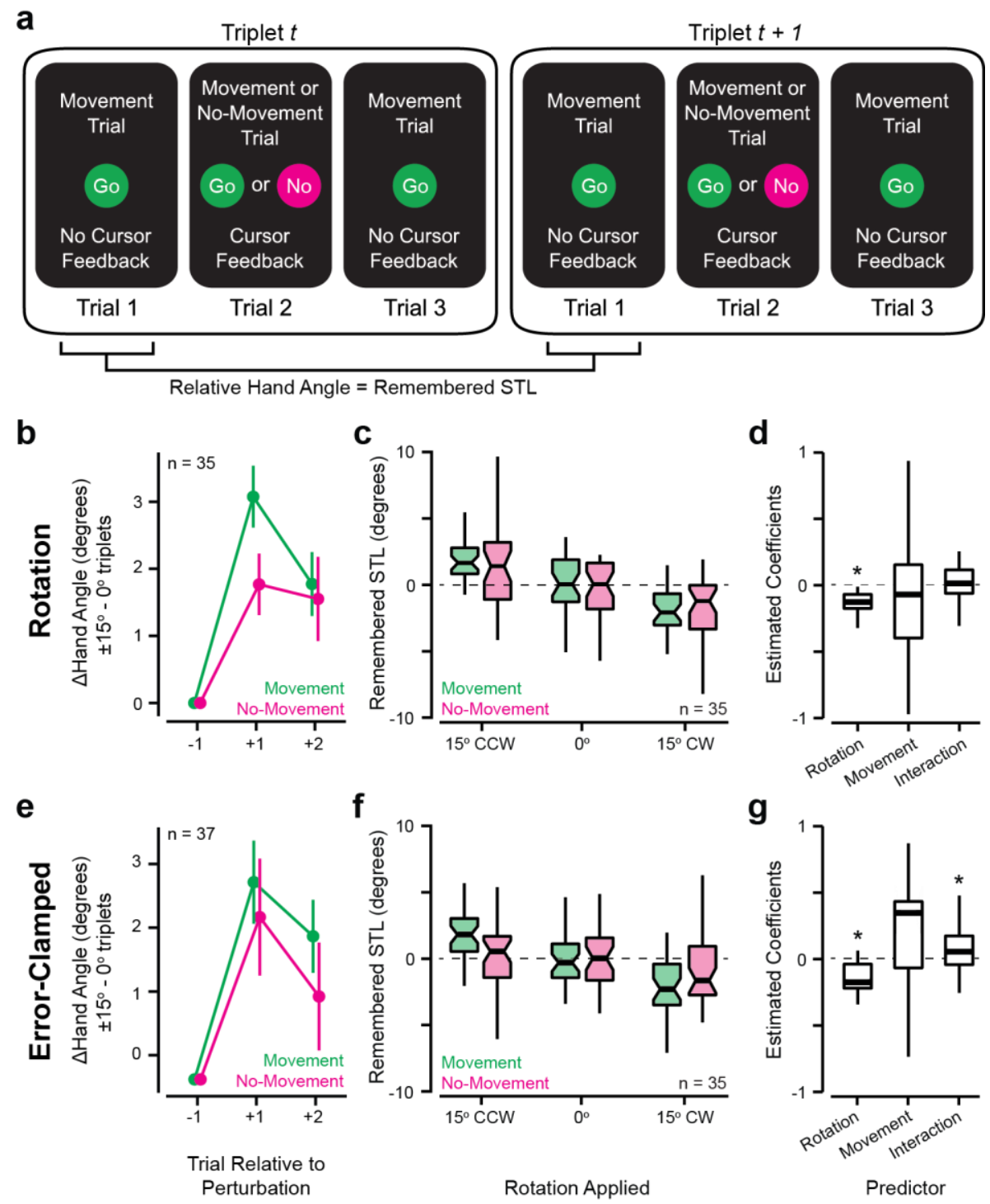

Supplementary Figure 1. Evidence that STL memories are maintained. (a) Schematic of the trial order in two consecutive triplets, illustrating that the 2 trials after each Movement/No-Movement trial are performed without visual feedback. (b) Change in hand angle relative to the first trial of the triplet in which the perturbation being considered occurs for Movement (green) and No-Movement (magenta) trials (remembered STL), for participants in an experiment where feedback was rotated relative to actual mouse position on Movement trials. Data are normalized to the change in hand angle observed on this trial on triplets with $0^{\circ}$ perturbations, and data are shown as mean \pm SEM. (c) Boxplots showing the distribution of participants' median persistent STL (change in hand angle relative to the pre-perturbation trial) two trials after the perturbation was observed. (d) Estimated coefficients from GLMs fitted to each participants' remembered STL performance with rotation applied, movement condition, and rotation:movement condition interaction provided as predictors. Wilcoxon's signed-rank tests showed statistically significant differences between rotation coefficients and $0\left(V=25, p_{a d j}=1.58 \times 10^{-7}, r=-0.80\right)$, but not between movement coefficients $\left(V=228, p_{a d j}=0.24\right)$ or rotation:movement interaction coefficients $\left(V=365, p_{a d j}=0.42\right)$ and 0 . (e-f) As in (b-d), but showing data collected in an experiment where participants saw error-clamped feedback on Movement trials. (g) Wilcoxon's signed-rank tests showed statistically significant differences 
interaction coefficients and $0\left(V=490, p_{a d j}=0.02, r=0.41\right)$, but not between movement coefficients $(V=$ 428, $\left.p_{a d j}=0.25\right)$ and 0. Boxplot center: median, notch: $95 \%$ confidence interval of the median, box edges: $1^{\text {st }}$ and $3^{\text {rd }}$ quartiles, whiskers: most extreme value within $1.5 *$ interquartile range of the median. Notches have been omitted for panels $\mathbf{d}$ and $\mathbf{g}$ because $95 \%$ confidence intervals exceeded the interquartile range. * indicates $p<0.05$.

a

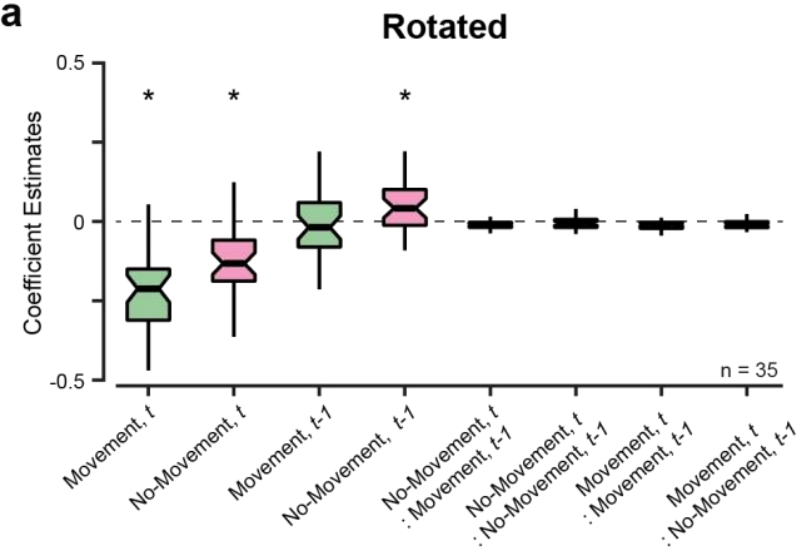

Coefficient Labels b

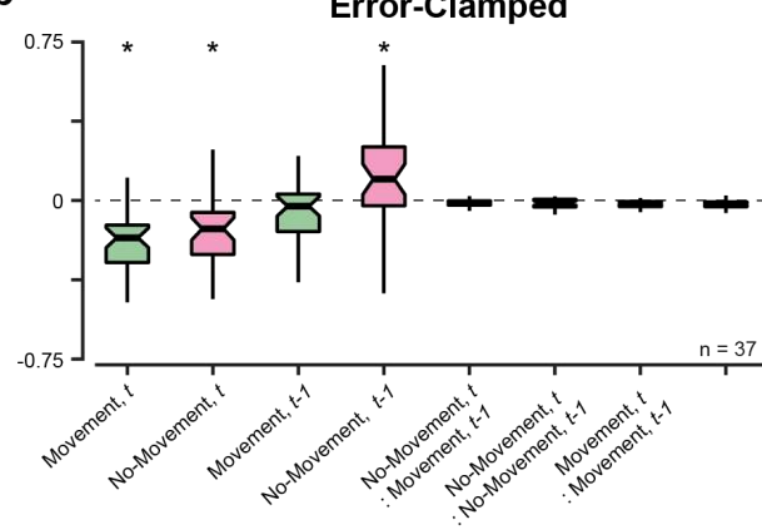

Coefficient Labels

Supplementary Figure 2. Coefficients from participant-wise GLMs with the perturbations applied on current and preceding Movement and No-Movement trials used as predictors. (a) Estimated coefficients from GLMs fit to each participant's single-trial learning (STL) measurements, using the perturbation applied on the current and preceding triplets as predictors. Participants were exposed to rotated cursor feedback on Movement trials. In the x-axis labels, $t$ indicates that the perturbation was applied on the current triplet, while $t-1$ indicates that the perturbation was applied on the preceding triplet. The distribution of coefficient estimates for Movement perturbations applied on the current triplet (Movement, $t$ ) was significantly different from 0 (Wilcoxon's signed-rank test, $V=16, p_{\text {adj }}=7.87 \times 10^{-8}, r=$ -0.83). The same was true for the coefficient estimates for No-Movement perturbations applied on the current triplet (No-Movement, $t ; V=74, p_{a d j}=9.50 \times 10^{-5}, r=-0.67$ ). No-Movement perturbations applied on the preceding triplet (No-Movement, $t-1)$ also differed from $0\left(V=463, p_{a d j}=0.04, r=0.40\right)$. Notably, No-Movement, $t-1$ coefficients tended to be positive (median: 0.05), suggesting that any effects of perturbations on previous No-Movement trials are not examples of residual adaptation from those trials, as any such effects would take on a negative coefficient. No other coefficient estimates differed significantly from 0 (Movement, $t-1: V=283, p_{a d j}=0.70$; No-Movement, $t$ : Movement, $t-1: V=265, p_{a d j}=0.80$; NoMovement, $t$ : No-Movement, $t-1: V=246, p_{a d j}=0.70$; Movement, $t$ : Movement, $t-1: V=254, p_{a d j}=0.70$; Movement, $t$ : No-Movement, $\left.t-1: V=235, p_{a d j}=0.70\right)$. (b) As in (a), except that participants were exposed to error-clamped cursor feedback on Movement trials. The distribution of coefficient estimates for Movement perturbations applied on the current triplet (Movement, $t$ ) was significantly different from 0 (Wilcoxon signedrank test, $\left.V=92, p_{a d j}=0.0002, r=-0.64\right)$. The same was true for the coefficient estimates for No-Movement perturbations applied on the current triplet (No-Movement, $t ; V=149, p_{\text {adj }}=0.008, r=-0.47$ ). No-Movement perturbations applied on the preceding triplet (No-Movement, $t-1)$ also differed from $0\left(V=576, p_{a d j}=\right.$ $0.002, r=0.56$ ). No other coefficient estimates differed significantly from 0 (Movement, $t-1: V=300, p_{a d j}=$ 0.71 ; No-Movement, $t$ : Movement, $t-1: V=248, p_{a d j}=0.87$; No-Movement, $t$ : No-Movement, $t-1: V=178$, $p_{a d j}=0.96$; Movement, $t:$ Movement, $t-1: V=240, p_{a d j}=0.87$; Movement, $t:$ No-Movement, $t-1: V=$ 
Implicit adaptation of withheld movements - 11

243, $\left.p_{\text {adj }}=0.32\right)$. Boxplot center: median, notches: $95 \%$ confidence interval of the median, edges: $1^{\text {st }}$ and $3^{\text {rd }}$ quartile, whiskers: most extreme datapoints within 1.5 IQR of the median. ${ }^{*}$ indicates $p_{a d j}<0.05$

To assess the potential similarity of mechanisms underlying adaptation after errors on Movement and No-Movement trials, we compared STL amplitude under each condition, reasoning that there should be a relationship if STL is supported by the same mechanisms on Movement and No-Movement trials. When we considered instances of STL in the direction that would compensate for the observed error (update opposite rotation, Supplementary Fig. 3a), within-subject changes in hand angle were correlated between Movement and No-Movement trials (Pearson's $r=0.45, p=0.007$; Supplementary Fig. 3b). Conversely, changes in hand angle in the direction that would exacerbate the observed error (update in direction of rotation, Supplementary Fig. 3a) were uncorrelated between Movement and No-Movement trials (Pearson's $r=0.03, p=0.86$, Supplementary Fig. 3c). Together, these observations suggest that the same learning process may underlie adaptive single-trial learning events in response to errors on both kinds of trials, while maladaptive changes in hand-angle may be attributable to potential sources of random noise. In spite of the correlation between the amplitudes of adaptive changes in hand angle on Movement and No-Movement trials, the overall amplitude of the changes in hand angle opposite the direction of the rotation were nonetheless smaller on No-Movement trials than on Movement trials (Wilcoxon's signed-rank test: $W=88, p=0.0002$, rank-biserial correlation $=0.14$ ), indicating that errors on No-Movement trials may not drive adaptation as strongly as errors on Movement trials. (We return to this issue in the Discussion.) 

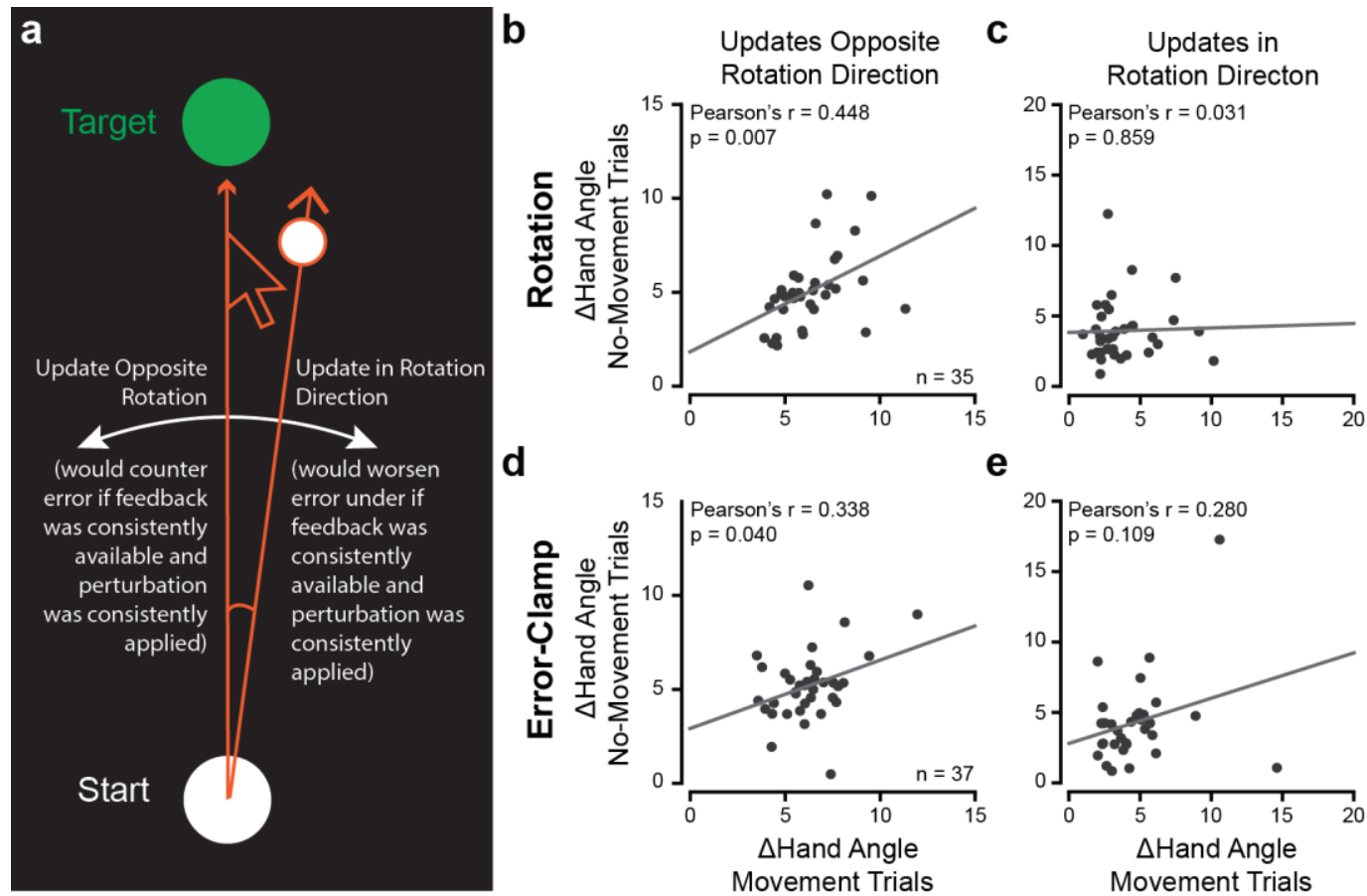

Supplementary Figure 3. Correlations between STL magnitude after Movement and No-Movement trials. (a) Schematic illustrating the implications of changes in hand angle (updates) in the same direction as or opposite direction to the perturbation applied. Under "normal" circumstances when we encounter a consistent perturbation, adaptation that causes the hand to move in the direction opposite the perturbation will counter that perturbation and tend to minimize future errors. (b) Scatter plot showing the relationship between individual subjects' STL amplitude in the direction opposite the rotation on Movement and NoMovement trials. Data were collected from an experiment where participants saw rotated feedback on Movement trials. (c) As in (b), but for STL amplitude in the same direction as the rotation applied. (d-e) As in $(\mathbf{b}-\mathbf{c})$, but for participants who received error-clamped feedback on Movement trials.

\section{Motor adaptation in response to errors during error clamp trials}

We note that rotated visual feedback on Movement trials was sensitive to people's actual reaching directions (i.e., the rotation was simply added to their natural reach direction, as is typical in visuomotor rotation tasks). Thus, it is possible that introducing these directional contingencies affected how people behaved in response to perceived errors. To rule this out, we recruited participants $(n=37)$ for a second experiment where the visual cursor moved in a fixed path ("error-clamped" feedback ${ }^{8}$ ) in one of three directions $\left(0^{\circ}\right.$ or $\left.15^{\circ} \mathrm{CW} / \mathrm{CCW}\right)$ on trials with feedback (Fig. 2e). In line with the effects of rotated cursor feedback, a LMM revealed main effects of error-clamped feedback $\left(F(2,1827.8)=79.46, p=2.2 \times 10^{-16}\right.$, partial $\left.R^{2}=0.08\right)$ and an interaction between rotation and movement condition $(F(2,1831.8)=8.45, p=$ 


$$
\text { Implicit adaptation of withheld movements }-13
$$

\section{Supplementary Table 2.}

Pairwise post-hoc comparisons between estimated marginal means in Fig. $2 \mathrm{~h}$

\begin{tabular}{|c|c|c|c|c|c|c|c|c|}
\hline \multicolumn{2}{|c|}{ Group 1} & \multicolumn{2}{|c|}{ Group 2} & \multirow{2}{*}{$\begin{array}{l}\text { Est. } \\
\text { Diff. }\end{array}$} & \multirow{2}{*}{$\mathrm{t}$} & \multirow{2}{*}{$d f$} & \multirow{2}{*}{$\begin{array}{c}\text { FDR- } \\
\text { adjusted } p\end{array}$} & \multirow{2}{*}{$\begin{array}{c}\text { Cohen's } \\
\text { d }\end{array}$} \\
\hline Trial Type & Rotation & Trial Type & Rotation & & & & & \\
\hline$M$ & $0^{\circ}$ & M & $15^{\circ} \mathrm{CW}$ & $3.54^{\circ}$ & 7.55 & 1827 & ${ }^{*} 3.08 \times 10^{-13}$ & 0.56 \\
\hline$M$ & $0^{\circ}$ & $M$ & $15^{\circ} \mathrm{CCW}$ & $2.57^{\circ}$ & 5.57 & 1828 & ${ }^{*} 8.84 \times 10^{-8}$ & 0.41 \\
\hline$M$ & $15^{\circ} \mathrm{CW}$ & $M$ & $15^{\circ} \mathrm{CCW}$ & $6.11^{\circ}$ & 13.14 & 1828 & ${ }^{*} 8.51 \times 10^{-37}$ & 0.97 \\
\hline No-M & $0^{\circ}$ & No-M & $15^{\circ} \mathrm{CW}$ & $1.81^{\circ}$ & 3.21 & 1830 & * 0.002 & 0.29 \\
\hline No-M & $0^{\circ}$ & No-M & $15^{\circ} \mathrm{CCW}$ & $1.29^{\circ}$ & 2.25 & 1832 & ${ }^{*} 0.03$ & 0.20 \\
\hline No-M & $15^{\circ} \mathrm{CW}$ & No-M & $15^{\circ} \mathrm{CCW}$ & $3.10^{\circ}$ & 5.46 & 1833 & ${ }^{*} 1.24 \times 10^{-7}$ & 0.49 \\
\hline$M$ & $0^{\circ}$ & No-M & $0^{\circ}$ & $0.08^{\circ}$ & 0.16 & 1846 & 0.87 & -- \\
\hline$M$ & $15^{\circ} \mathrm{CW}$ & No-M & $15^{\circ} \mathrm{CW}$ & $1.81^{\circ}$ & 3.49 & 1846 & * 0.0009 & 0.29 \\
\hline$M$ & $15^{\circ} \mathrm{CCW}$ & No-M & $15^{\circ} \mathrm{CCW}$ & $1.19^{\circ}$ & 2.29 & 1846 & ${ }^{*} 0.03$ & 0.19 \\
\hline
\end{tabular}

Note. Abbreviations: M - Movement; No-M - No-Movement; Est. Diff. - Estimated Differences in degrees. Note that degrees of freedom pertain to the inputs to the model and are estimated using the

Kenward-Rogers approach. Statistically significant $p$-values are indicated with asterisks. ${ }^{*} p<0.05$.

Like participants who observed rotated cursor feedback on Movement trials, participants who

0.0002, partial $\left.R^{2}=0.0003\right)$, although there was no main effect of movement condition $(F(1,1844.1)=0.60$, $p=0.44$; Fig. 2h). Post-hoc comparisons of the model's estimated marginal means (Supplementary Table 2) revealed significant $S T L$ in response to non-zero error-clamped feedback on both Movement $\left(0^{\circ}\right.$ vs $15^{\circ}$ CW: $t(1827)=7.55, p_{a d j}=3.08 \times 10^{-13}$, Cohen's $d=0.56 ; 0^{\circ}$ vs $15^{\circ} \mathrm{CCW}: t(1828)=5.57, p_{a d j}=8.84 \times 10^{-}$ ${ }^{8}$, Cohen's $\left.d=0.41\right)$ and No-Movement trials $\left(0^{\circ}\right.$ vs $15^{\circ} \mathrm{CW}: t(1830)=3.21, p_{a d j}=0.002$, Cohen's $d=0.29$; $0^{\circ}$ vs $15^{\circ} \mathrm{CCW}: t(1832)=2.25, p_{a d j}=0.03$, Cohen's $\left.d=0.20\right)$. Adaptation in the presence of a $15^{\circ}$ errorclamp was significantly greater on Movement trials than No-Movement trials for CW $\left(t(1846)=3.50, p_{a d j}=\right.$ 0.0009 , Cohen's $d=0.29)$ and CCW clamps $\left(t(1846)=2.29, p_{a d j}=0.03\right.$, Cohen's $\left.d=0.19\right)$. observed error-clamped cursor feedback on Movement trials showed persistent STL beyond the triplet in 
Implicit adaptation of withheld movements - 14

which the error was observed (remembered STL, Supplementary Fig. 1e-f), and participant-wise GLMs' fitted rotation coefficients differed significantly from 0 (Supplementary Fig. 1g; Wilcoxon's signed-rank test; $\left.V=31, p_{a d j}=1.04 \times 10^{-7}, r=-0.80\right)$. Additionally, STL for error-clamp participants was not attributable to errors observed on preceding Movement trials (Supplementary Fig. 2b). Unlike the rotation study participants, error-clamp study participants' rotation:movement interaction coefficients also differed from 0 $\left(V=490, p_{a d j}=0.02, r=0.41\right)$, suggesting that they showed less remembered STL after No-Movement trials than Movement trials. Nonetheless, these observations indicate that changing the nature of feedback on Movement trials did not eliminate the capacity of errors on No-Movement trials to induce genuine learning.

counter to the error observed was correlated between Movement and No-Movement conditions (Pearson's $r=0.34, p_{a d j}=0.04$ ), while the amplitude of STL events that proceeded in the same direction as the presented error was not correlated between Movement and No-Movement conditions $\left(r=0.28, p_{a d j}=0.11\right)$. Thus, these data generally replicate the rotation study, strengthening the claim that the same learning process may underlie adaptive single-trial learning events in response to errors on both Movement and NoMovement trials.

Adaptation on No-Movement trials does not depend on within-session adaptation on Movement trials

In two further experiments, we asked if adaptation to errors in the No-Movement condition was contingent on sharing a context with the Movement condition. That is, does learning in the No-Movement condition only occur if it happens alongside similar learning in the Movement condition? If so, this would suggest that adaptive responses in the No-Movement condition may reflect a "cueing" effect, whereby an adapted sensorimotor map is cued by the visual error and then retrieved on the subsequent trial(s). While our retention (Supplementary Figs. 1-2) and correlation (Supplementary Fig. 3) results argue against this interpretation, we directly tested this in another pair of experiments. Here, we only included $0^{\circ}$ rotated (Fig. 3a) or clamped (Fig. 3d) error feedback on Movement trials, and the normal mix of $0^{\circ}, 15^{\circ} \mathrm{CW} / \mathrm{CCW}$ errors on the No-Movement trials. The key results were replicated - learning was preserved in the NoMovement condition even when error feedback had never been associated with executed movements (Fig. 
3b - c, LMM: $F(557) 23.01, p=2.07 \times 10^{-6}$, partial $R^{2}=0.04, \mathbf{e}-\mathbf{f}, F(1456)=11.63, p=9.76 \times 10^{-6}$, partial

$R^{2}=0.02$ ). Post-hoc pairwise comparisons showed that adaptation was significantly different between

triplets with clockwise and counterclockwise errors for both the rotation $\left(t(557)=4.80, p=2.07 \times 10^{-6}\right.$,

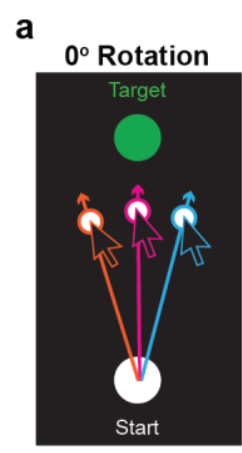

b

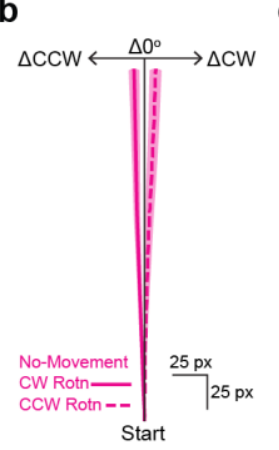

e

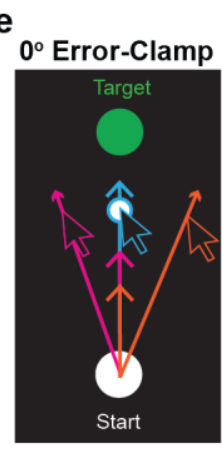

Go Trials with Feedback f

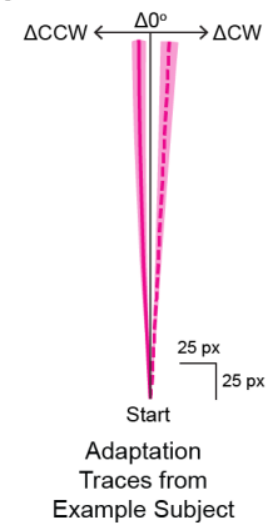

c

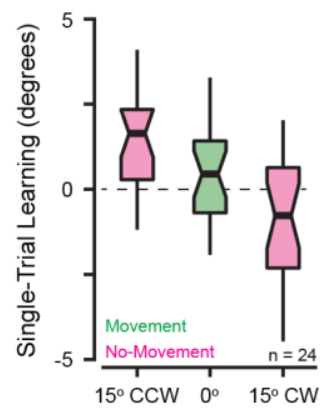

g

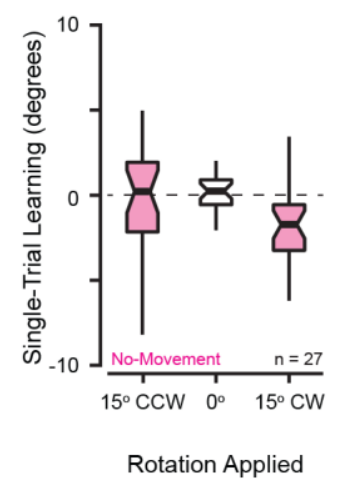

d

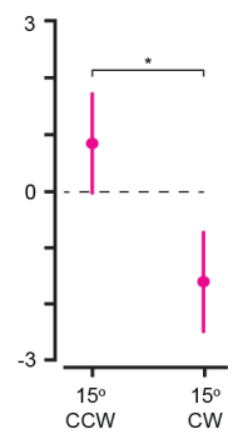

h

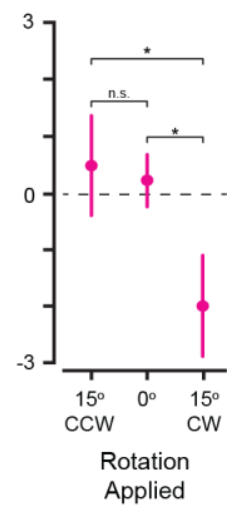

Figure 3. Single-trial learning on No-Movement trials when feedback on Movement trials underwent (b) An example participant's mean \pm SEM changes in reach paths across triplets with a rotation applied. Green shading indicates that data are from triplets in which a perturbation was applied on a Movement trial, while magenta shading indicates that the perturbation was applied on a No-Movement trial. Solid lines indicate that a clockwise (CW) rotation was applied on the relevant perturbation trial, while dashed lines indicate that a counterclockwise (CCW) rotation was applied. (c) Single-trial learning in response to different directions of simulated errors on No-Movement trials (magenta) and no rotation on Movement trials (green). (d) Estimated marginal means $\pm 95 \%$ confidence intervals from the linear mixed models (LMM) fit to each participant's single-trial learning performance summarized in (c). (e-h) As (a-f), but for participants in an experiment with $0^{\circ}$ error-clamped feedback on Movement trials. Note that data in $(\mathbf{g})$ from the $0^{\circ}$ rotation applied condition are collapsed across Movement and No-Movement trials. Asterisks indicate statistically significant differences between post-hoc comparisons of estimated marginal means. Please refer to Supplementary Table 3 for detailed statistical results. Boxplot centers: median, notches: $95 \%$ confidence 


$$
\text { Implicit adaptation of withheld movements }-16
$$

354

355

356

357

358

interval of the median, box edges: $1^{\text {st }}$ and $3^{\text {rd }}$ quartiles, whiskers: most extreme values within $1.5^{*} \mathrm{IQR}$ of the median.

\section{Supplementary Table 3.}

Pairwise post-hoc comparisons between estimated marginal means in Fig. 3

\begin{tabular}{|c|c|c|c|c|c|c|c|c|}
\hline \multicolumn{2}{|c|}{ Group 1} & \multicolumn{2}{|c|}{ Group 2} & \multirow{2}{*}{$\begin{array}{l}\text { Est. } \\
\text { Diff. }\end{array}$} & \multirow{2}{*}{$\mathrm{t}$} & \multirow{2}{*}{$\mathrm{df}$} & \multirow{2}{*}{$\begin{array}{c}\text { FDR- } \\
\text { adjusted } p\end{array}$} & \multirow{2}{*}{$\begin{array}{c}\text { Cohen's } \\
\mathrm{d}\end{array}$} \\
\hline Trial Type & Rotation & Trial Type & Rotation & & & & & \\
\hline \multicolumn{9}{|c|}{$0^{\circ}$ Rotation Applied on Movement Trials } \\
\hline No-M & $15^{\circ} \mathrm{CCW}$ & No-M & $15^{\circ} \mathrm{CW}$ & $2.45^{\circ}$ & 4.80 & 557 & ${ }^{*} 2.07 \times 10^{-6}$ & 0.40 \\
\hline \multicolumn{9}{|c|}{$0^{\circ}$ Error-Clamped Feedback on Movement Trials } \\
\hline No-M & $15^{\circ} \mathrm{CCW}$ & No-M & $15^{\circ} \mathrm{CW}$ & $2.47^{\circ}$ & 4.03 & 1453 & * 0.001 & 0.40 \\
\hline No-M & $15^{\circ} \mathrm{CW}$ & $M+N o-M$ & $0^{\circ}$ & $2.21^{\circ}$ & 4.64 & 1457 & ${ }^{*} 1.16 \times 10^{-5}$ & 0.36 \\
\hline No-M & $15^{\circ} \mathrm{CCW}$ & $M+$ No-M & $0^{\circ}$ & $0.26^{\circ}$ & 0.56 & 1456 & 0.57 & -- \\
\hline
\end{tabular}

Note. Abbreviations: M - Movement; No-M - No-Movement; Est. Diff. - Estimated Differences in degrees. Note that degrees of freedom pertain to the inputs to the model and are estimated using the Kenward-Rogers approach. Statistically significant $p$-values are indicated with asterisks. ${ }^{*} p<0.05$.

Although these results demonstrated that sharing a context with movement-accompanied adaptation was not a necessary condition for No-Movement adaptation, we did observe attenuated learning on No-Movement Trials: Adaptation in response to errors on both Movement and No-Movement trials was greater in experiments in which non-zero rotations and clamps were applied on Movement trials (Supplementary Fig. 4a, two-sample t-test: $t(85)=5.94, p=6.15 \times 10^{-8}$, Cohen's $d=1.15$ ). Crucially, STL across all four of our experiments was within the range of learning rates observed in previous studies with identical error sizes (Supplementary Fig. 5).

Prior work has indicated that subjects' within-session histories of error affect the amplitude of motor adaptation, offering a potential explanation of the observed differences. ${ }^{18-20}$ Here, differences in the experimental designs mandated by the need to counterbalance the number of Movement and No-

371 Movement trials within a session may have affected the prevalence of different kinds of errors and error 
transitions in the experiments described in Figs. 2-3, potentially influencing the amplitude of STL observed within each experiment.

Following the analyses in (ref. ${ }^{20}$ ), we first investigated whether observation of two successive errors in opposite directions decreased sensitivity to error and may have accounted for the differences in overall STL amplitude between the experiments contributing to Figs. 2-3. To our surprise, in each experiment, we observed an increase in STL after a change in the direction of the perturbation relative to STL after maintaining the direction of the perturbation (Supplementary Fig. 4b): Across all experiments, when the direction of the observed error was maintained between triplets, STL was significantly smaller than STL when an error abruptly emerged (i.e., when a non-zero error occurred after a triplet with a $0^{\circ}$ perturbation trial; $t(113)=2.18, p_{\text {adj }}=0.047$, Cohen's $\left.d=0.29\right)$, or when the error's direction changed $(t(109)$ $=2.38, p_{a d j}=0.047$, Cohen's $d=0.32$ ). These data suggest that, when participants are exclusively exposed to triplets with randomly varying error, inconsistent errors may increase the rate of implicit adaptation, in contrast to what was previously reported in a blocked learning design..$^{20}$ We note that participants in experiments where only $0^{\circ}$ perturbations were applied on Movement trials were exposed to significantly fewer instances of inconsistent errors than participants who experienced $0^{\circ}$ and $15^{\circ}$ perturbations on Movement trials (Supplementary Fig. 4d, two-sample Kolmogorov-Smirnov tests; Rotation $0^{\circ} / 15^{\circ}$ vs Rotation $0^{\circ}$ only on Movement Trials: $D=1, p_{\text {adj }}=1.29 \times 10^{-12}$; Error-clamp $0^{\circ}, 15^{\circ} \mathrm{vs}$ Error-clamp $0^{\circ}$ only on Movement Trials: $\left.D=1, p_{a d j}=1.66 \times 10^{-13}\right)$. Taken together, these observations suggest that overall STL was smaller in experiments where participants only saw $0^{\circ}$ perturbations on Movement trials because of decreases in error sensitivity arising from a paucity of inconsistent error transitions.

In addition to effects of error direction transitions, prior studies have also reported putative effects of reward on implicit adaptation. ${ }^{22-24}$ Specifically, when unexpected feedback deviations still result in task success (i.e., the cursor movement terminates within the target), implicit adaptation is attenuated. When we retrospectively considered the proportion of trials where the visually displayed cursor landed completely on target and may have satisfied the implicit task goal of "hitting" the target, we found that participants who experienced exclusively $0^{\circ}$ error-clamped feedback on Movement trials saw the cursor hit the target $>25 \%$ more than participants in the other experiments (Supplementary Fig. 4e). This greater rate of apparent 
task success may have established a context that suppressed adaptation in a manner consistent with prior work. 22,24

Together, these data suggest that the order and content of trials and the nature of the stimuli employed in the rotation and Error Clamp experiments without non-zero perturbations on Movement trials effectively reduced learning rates in these experiments and may account for the lower observed levels of adaptation between the two sets of studies. Overall, these experiments support our hypothesis that, in the right circumstances, motor adaptation can proceed without movement execution.

a

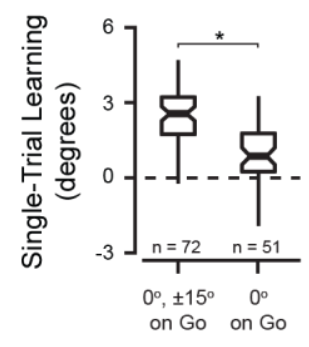

b

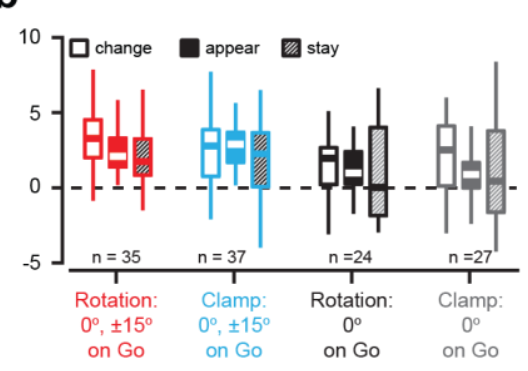

C

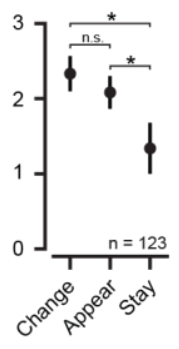

d
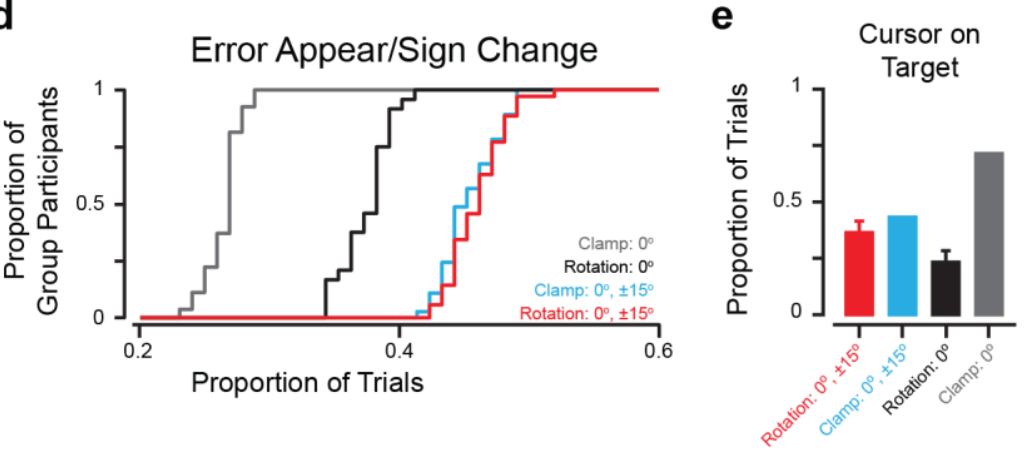

Supplementary Figure 4. Effects of changes in error sign on learning rate and graphics showing details of experiments' trial composition. (a) Boxplots showing STL observed across Movement and No-Movement trials in the experiments shown in Fig. $2\left(0^{\circ}, \pm 15^{\circ}\right.$ on Go) and the experiments shown in Fig. 3 ( $0^{\circ}$ on Go). A two-sample t-test revealed a significant difference between STL amplitude in each kind of experiment $\left(t(85)=5.94, p=6.15 \times 10^{-8}\right.$, Cohen's $\left.d=1.15\right)$. (b) Boxplots showing STL observed on triplets where the perturbation direction changed relative to the previous triplet (open boxes), triplets where a nonzero perturbation occurred after a $0^{\circ}$ perturbation (filled boxes), and triplets where the perturbation direction was the same as the previous triplet (hashed boxes). (c) STL across all experiments in the present study, divided by the type of error transition observed. Data are shown as mean \pm SEM. Paired t-tests between STL amplitudes between error transition conditions showed significant differences between triplets where error direction remained the same and triplets where errors appeared following a $0^{\circ}$-perturbation trial $(t(113)$ $=2.18, p_{\text {adj }}=0.047$, Cohen's $\left.d=0.29\right)$ and triplets where the direction of the perturbation changed $(t(109)$ $=2.38, p_{a d j}=0.047$, Cohen's $d=0.32$ ). There was no significant difference between triplets where error 
appeared following a $0^{\circ}$-perturbation triplet and triplets where the direction of the perturbation changed $\left(t(117)=0.43, p_{a d j}=0.67\right)$. (d) Cumulative distribution plots showing the proportion of participants with a certain proportion of trials or lower on which an error appeared or the sign of the perturbation changed between triplets. Two-sample Kolmogorov-Smirnov tests revealed that the proportion of trials where errors appeared or the perturbation sign changed was significantly lower for participants who received $0^{\circ}$-errorclamp perturbations on all Movement trials than for participants who received $0^{\circ}$-rotation perturbations on all Movement trials $\left(D=1, p_{\text {adj }}=2.21 \times 10^{-11}\right)$, who received $0 \% \pm 15^{\circ}$ rotation perturbations on all Movement trials $\left(D=1, p_{a d j}=2.31 \times 10^{-13}\right)$, and who received $0^{\circ} \pm 15^{\circ}$ error-clamp perturbations on all Movement trials $\left(D=1, p_{a d j}=1.66 \times 10^{-13}\right)$. The proportion of trials where errors appeared/changed for participants who received $0^{\circ}$-rotation perturbations on all Movement trials was also significantly lower that that observed for participants who received $0^{\circ} \pm 15^{\circ}$ rotation perturbations on all Movement trials $\left(D=1, p_{\text {adj }}=1.29 \times 10^{-12}\right)$, and who received $0^{\circ} \pm 15^{\circ}$ error-clamp perturbations on all Movement trials $\left(D=1, p_{a d j}=2.72 \times 10^{-14}\right)$. The proportion of trials where errors appeared/changed directions did not differ between participants who received $0 \% \pm 15^{\circ}$ rotation or error-clamp perturbations on all Movement trials $\left(D=0.21, p_{a d j}=0.42\right)$. (e) Bar plots showing the proportion of feedback trials on which the cursor landed completely on the target. The number of trials on which the cursor hit the target was inflated in the Clamp: $0^{\circ}$ study, because 1) all Movement trials were $0^{\circ}$ error-clamped trials, 2) there were as many Movement trials as No-Movement trials, and 3) there were also No-Movement trials showing $0^{\circ}$ simulated errors. The number of trials on which the cursor hit the target was deflated in the Rotation: $0^{\circ}$ study because there was no No-Movement trials showing $0^{\circ}$ simulated errors (i.e., there was no subset of trials on which the cursor was guaranteed to hit the target for these participants). Data are shown as mean \pm SEM when values varied between participants (rotation experiments) and as mean only when values did not vary (clamp experiments). Boxplot centers: median, notches: $95 \%$ confidence interval of the median, box edges: $1^{\text {st }}$ and $3^{\text {rd }}$ quartiles, whiskers: most extreme values within $1.5^{\star} \mathrm{IQR}$ of the median. ${ }^{*}$ indicates $p_{\mathrm{adj}}<0.05$.

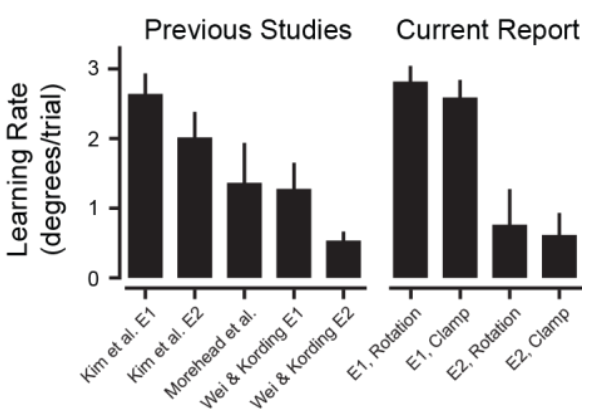

Supplementary Figure 5. Learning rates observed in the literature and the current study. Learning rates observed in previous studies are shown at left, and learning rates observed in each experiment in the current report are shown at the right. Data are shown as mean \pm SEM, and are shown for rotations/error clamp manipulations of $15^{\circ}$, with the exception of Wei \& Kording E2, where an $11^{\circ}$ perturbation was applied. Papers referred to and their corresponding reference numbers: Kim et al., 201814; Morehead et al., 20178; Wei \& Kording, 200921. "E1" in the current report refers to conditions where participants saw $0-15^{\circ}$ perturbations on Movement trials, and "E2" in the current report refers to conditions where participants saw only $0^{\circ}$ perturbations on Movement trials. Abbreviations: $E$, experiment. 


$$
\text { Implicit adaptation of withheld movements }-20
$$

\section{DISCUSSION}

The results presented above demonstrate that movements can be implicitly refined even when they are not performed. Participants who were cued to perform a movement towards a target but suppressed that movement after observation of a No-Go cue showed consistent, robust single-trial learning in response to an animation of erroneous cursor movement (Fig. 2b-d, f-h; Fig. 3b-d, f-h). This learning carried the key signatures of implicit adaptation of an internal model. Our findings support the fundamental assumptions of predictive processing frameworks of motor adaptation, where precise sensory predictions are generated from a movement intent (or "plan", "goal") and compared against sensory observations to induce errorbased learning. ${ }^{12,25}$

We argue that we have measured learning via an implicit process, and, by extension, that implicit adaptation of specific movements can proceed even when those movements are not performed. While visuomotor learning tasks do sometimes recruit cognitive learning strategies (e.g., the deliberate "re-aiming" of movements), multiple factors indicate that our studies measured implicit adaptation. ${ }^{7,26,27}$ First, in line with previous work isolating the implicit process, participants were instructed to ignore the visually displayed cursor and try to contact the target on every trial, a simple technique which has proven consistently effective at eliminating the explicit re-aiming of movements. . $^{8,13,14,28}$ Second, in addition to instructing participants in a manner that should have suppressed recruitment of explicit processes, the randomization of both the presence and direction of visual errors discourages explicit learning, reducing any motivation that participants may have had to apply ineffective re-aiming strategies $\left(\mathrm{see}^{29}\right)$. Third, we excluded data from any participants who did not appear to understand or recall our instruction to always aim at the target (see Methods), decreasing the likelihood that strategic re-aiming contaminated the analyzed data sets (however, we note that our key results did not change if these participants were included). Fourth, adaptation persisted into subsequent no-feedback trials (Supplementary Fig. 1b-g), a finding that is consistent with lingering implicit motor learning; it is unlikely that strategies would be maintained across trials where no feedback is present. Lastly, the magnitude of single-trial learning we observed was in line with multiple previous studies designed to measure implicit trial-by-trial motor adaptation (Supplementary Fig. 5). ${ }^{8,14,21}$ Our data thus provide striking evidence that internal forward models predict the state of the world following action 
Implicit adaptation of withheld movements -21

487

488

489

490

491

492

493

494

495

496

497

selection, instead of predicting the sensory consequences of movement per se, and that this prediction is then compared with observed sensory events to compute a sensory-prediction error to drive learning.

We also note that the adaptation effects observed in the No-Movement conditions were not attributable to a recall process driven by movement-based learning. That is, No-Movement adaptation was not related to errors on preceding Movement triplets (Supplementary Fig. 2) and proceeded independently of visual errors being present on Movement trials (Fig. 3). While overall levels of adaptation were smaller during the two experiments with no perturbations on Movement trials, this reduced level of adaptation was likely attributable to features of the experimental design: The decreased likelihood of errors and changes in trial-by-trial error variance in these experiments may have reduced learning rates (Supplementary Fig.

4). Additionally, an increased likelihood of task success (hitting the target) during the error-clamp study with no perturbations on Movement trials may have further suppressed learning rates. ${ }^{22,24}$

Our observation that changes in the direction of the perturbation enhanced the degree of singletrial adaptation (Supplementary Fig. 4b) appears to conflict with a recently published report suggesting that changes in the direction of error decrease error sensitivity and accompanying learning rate. ${ }^{20}$ However, several differences between this study and the present study may account for these differences. For instance, the experiments described in ${ }^{20}$ varied perturbation amplitude, but held perturbation sign consistent, whereas we varied perturbation sign. Additionally, we observed effects of changes in the sign of the perturbation applied on STL, while ${ }^{20}$ reported effects of changes in the sign of the visual error observed on cumulative learning across sessions. Finally, in our study, changes in errors took place across triplets (sets of 3 trials); in contrast, in the experiments in ${ }^{20}$ errors may have changed between single trials. It may be that a different mechanism for tracking and adjusting error sensitivity in response to environmental variability is recruited when visual feedback is not present on all trials, or when the sign in addition to the magnitude of the perturbation varies.

While our results demonstrate that overt movement is not necessary for motor adaptation, we are not able to discern whether the generation of a descending motor command is a necessary component for updating the forward model. To reliably elicit prediction generation from the forward model, we employed a motor inhibition paradigm, briefly cueing participants to reach before presenting them with an abrupt stop signal. This strategy is likely to have recruited mechanisms of motor inhibition beyond the primary motor 
Implicit adaptation of withheld movements -22

cortex, leaving open the possibility that a descending motor command and not an upstream motor plan per se provide the necessary input for forward internal model adaptation. ${ }^{30-32}$ Future experimentation quantifying neural (EEG) or physiological (EMG) signals during this task and measuring their relationship with adaptation on No-Movement trials will be crucial for addressing this point. Inspired by work in nonhuman primates, we speculate that shared motor cortical preparatory states in the Movement and NoMovement conditions may produce comparable inputs to downstream internal models. ${ }^{33}$

In addition to suggesting that motor planning is sufficient for motor adaptation, our data also demonstrate that errors paired with movements support a greater degree of learning than errors without movement, as participants showed significantly stronger STL over triplets with Movement trials than NoMovement trials. This may suggest that movement provides additional training input to the forward model beyond what erroneous visual feedback provides alone. Alternately (though not mutually exclusive), it may be that the absence of movement-related proprioceptive feedback dampens the sensory prediction error signals that drive adaptation, perhaps by failing to counterbalance inhibitory signals predicting proprioceptive feedback. ${ }^{1,34} \mathrm{~A}$ simpler set of explanations are that participants simply did not recognize the Go cue to set a movement goal and engage the forward model on a subset of No-Movement trials, or that the timing of the simulated error was too variable, relative to the internal prediction, to elicit maximal adaptation. Further experimentation will be required to address these possibilities. Nonetheless, our data demonstrate the significant influence of the brain's prediction signals on learning - even without the ability to directly attribute sensory feedback to an actual movement, the brain's prediction of a planned movement's sensory consequences support the error computations that adapt future behavior.

We note that other forms of learning also appear to occur without overt task execution. First, motor learning can occur through observation of another individual learning the same task or observation of target movement without active tracking - the degree to which such learning reflects action selection processes or implicit motor adaptation (or both) is unclear, though our current results may support observation-based implicit motor adaptation. ${ }^{35,36}$ Outside of motor learning, fear associations can be extinguished by instructing participants to imagine a fear-predicting stimulus even when they are not presented with the stimulus, with this "imagination" protocol generating neural signatures of the negative prediction errors observed during naturalistic fear extinction. ${ }^{37,38}$ Considering this prior work and the findings presented in this study, it may 
bioRxiv preprint doi: https://doi.org/10.1101/2021.08.12.456140; this version posted August 13, 2021. The copyright holder for this preprint (which was not certified by peer review) is the author/funder, who has granted bioRxiv a license to display the preprint in perpetuity. It is made available under aCC-BY 4.0 International license.

$$
\text { Implicit adaptation of withheld movements }-23
$$

543 be that the generation of predictions for comparison with subsequent sensory feedback is sufficient for

544 error-based learning across motor and non-motor domains alike. In other words, task execution may not

545 always be required for learning, so long as the prediction and sensory feedback needed to compute error

546 are present. 
Implicit adaptation of withheld movements - 24

\section{References}

1. Miall, R. C. \& Wolpert, D. M. Forward Models for Physiological Motor Control. Neural Networks 9, 1265-1279 (1996).

2. Jordan, M. I. \& Rumelhart, D. E. Forward Models: Supervised Learning with a Distal Teacher. Cognitive Science 16, 307-354 (1992).

3. Wallman, J. \& Fuchs, A. F. Saccadic Gain Modification: Visual Error Drives Motor Adaptation. Journal of Neurophysiology 80, 2405-2416 (1998).

4. Shadmehr, R., Smith, M. A. \& Krakauer, J. W. Error Correction, Sensory Prediction, and Adaptation in Motor Control. Annu. Rev. Neurosci. 33, 89-108 (2010).

5. Tseng, Y., Diedrichsen, J., Krakauer, J. W., Shadmehr, R. \& Bastian, A. J. Sensory Prediction Errors Drive Cerebellum-Dependent Adaptation of Reaching. Journal of Neurophysiology 98, 54-62 (2007).

6. Mazzoni, P. \& Krakauer, J. W. An Implicit Plan Overrides an Explicit Strategy during Visuomotor Adaptation. J. Neurosci. 26, 3642-3645 (2006).

7. Taylor, J. A., Krakauer, J. W. \& Ivry, R. B. Explicit and Implicit Contributions to Learning in a Sensorimotor Adaptation Task | Journal of Neuroscience. The Journal of Neuroscience 34, (2014).

8. Morehead, J. R., Taylor, J. A., Parvin, D. E. \& Ivry, R. B. Characteristics of Implicit Sensorimotor Adaptation Revealed by Task-irrelevant Clamped Feedback. J Cogn Neurosci 29, 1061-1074 (2017).

9. Kilteni, K., Andersson, B. J., Houborg, C. \& Ehrsson, H. H. Motor imagery involves predicting the sensory consequences of the imagined movement. Nat Commun 9, 1617 (2018).

10. Voss, M., Ingram, J. N., Haggard, P. \& Wolpert, D. M. Sensorimotor attenuation by central motor command signals in the absence of movement. Nat Neurosci 9, 26-27 (2006).

11. Sheahan, H. R., Ingram, J. N., Žalalytė, G. M. \& Wolpert, D. M. Imagery of movements immediately following performance allows learning of motor skills that interfere. Sci Rep 8, 14330 (2018).

12. Sheahan, H. R., Franklin, D. W. \& Wolpert, D. M. Motor Planning, Not Execution, Separates Motor Memories. Neuron 92, 773-779 (2016).

13. Tsay, J. S., Parvin, D. E. \& Ivry, R. B. Continuous reports of sensed hand position during sensorimotor adaptation. Journal of Neurophysiology 124, 1122-1130 (2020).

14. Kim, H. E., Morehead, J. R., Parvin, D. E., Moazzezi, R. \& Ivry, R. B. Invariant errors reveal limitations in motor correction rather than constraints on error sensitivity. Communications Biology 1 , 1-7 (2018).

15. Avraham, G., Morehead, J. R., Kim, H. E. \& Ivry, R. B. Reexposure to a sensorimotor perturbation produces opposite effects on explicit and implicit learning processes. PLOS Biology 19, e3001147 (2021).

16. Poh, E., Al-Fawakari, N., Tam, R., Taylor, J. A. \& McDougle, S. D. Generalization of motor learning in psychological space. bioRxiv 2021.02.09.430542 (2021) doi:10.1101/2021.02.09.430542.

17. Hadjiosif, A. M., Krakauer, J. W. \& Haith, A. M. Did We Get Sensorimotor Adaptation Wrong? Implicit Adaptation as Direct Policy Updating Rather than Forward-Model-Based Learning. J. Neurosci. 41, 2747-2761 (2021).

18. Wei, K. \& Koerding, K. Uncertainty of feedback and state estimation determines the speed of motor adaptation. Front. Comput. Neurosci. 0, (2010). 
Implicit adaptation of withheld movements - 25

587

588

589

590

591

592

593

594

595

596

597

598

599

600

601

602

603

604

605

606

607

608

609

610

611

612

613

614

615

616

617

618

619

620

621

622

623

624

625

19. Semrau, J. A., Daitch, A. L. \& Thoroughman, K. A. Environmental experience within and across testing days determines the strength of human visuomotor adaptation. Exp Brain Res 216, 409-418 (2012).

20. Albert, S. T. et al. An implicit memory of errors limits human sensorimotor adaptation. Nat Hum Behav 5, 920-934 (2021).

21. Wei, K. \& Körding, K. Relevance of Error: What Drives Motor Adaptation? Journal of Neurophysiology 101, 655-664 (2009).

22. Kim, H. E., Parvin, D. E. \& Ivry, R. B. The influence of task outcome on implicit motor learning. eLife 8, e39882 (2019).

23. Galea, J. M., Mallia, E., Rothwell, J. \& Diedrichsen, J. The dissociable effects of punishment and reward on motor learning. Nat Neurosci 18, 597-602 (2015).

24. Leow, L.-A., Marinovic, W., Rugy, A. de \& Carroll, T. J. Task errors contribute to implicit aftereffects in sensorimotor adaptation. European Journal of Neuroscience 48, 3397-3409 (2018).

25. Franklin, D. W. \& Wolpert, D. M. Computational Mechanisms of Sensorimotor Control. Neuron 72 , 425-442 (2011).

26. Krakauer, J., W., Hadjiosif, A., Xu, J. \& Wong, A., L. Motor Learning. in Comprehensive Physiology (ed. Terjung, R.) (Wiley, 2019). doi:10.1002/cphy.

27. McDougle, S. D. \& Taylor, J. A. Dissociable cognitive strategies for sensorimotor learning. Nat Commun 10, 40 (2019).

28. McDougle, S. D., Ivry, R. B. \& Taylor, J. A. Taking Aim at the Cognitive Side of Learning in Sensorimotor Adaptation Tasks. Trends in Cognitive Sciences 20, 535-544 (2016).

29. Hutter, S. A. \& Taylor, J. A. Relative sensitivity of explicit reaiming and implicit motor adaptation. Journal of Neurophysiology 120, 2640-2648 (2018).

30. Xu, K. Z. et al. Neural Basis of Cognitive Control over Movement Inhibition: Human fMRI and Primate Electrophysiology Evidence. Neuron 96, 1447-1458.e6 (2017).

31. Osada, T. et al. An Essential Role of the Intraparietal Sulcus in Response Inhibition Predicted by Parcellation-Based Network. J. Neurosci. 39, 2509-2521 (2019).

32. Duque, J., Greenhouse, I., Labruna, L. \& Ivry, R. B. Physiological Markers of Motor Inhibition during Human Behavior. Trends in Neurosciences 40, 219-236 (2017).

33. Vyas, S. et al. Neural Population Dynamics Underlying Motor Learning Transfer. Neuron 97, 11771186.e3 (2018).

34. Requarth, T., Kaifosh, P. \& Sawtell, N. B. A Role for Mixed Corollary Discharge and Proprioceptive Signals in Predicting the Sensory Consequences of Movements. J. Neurosci. 34, 16103-16116 (2014).

35. Mattar, A. A. G. \& Gribble, P. L. Motor Learning by Observing. Neuron 46, 153-160 (2005).

36. Cain, M., Botschko, Y. \& Joshua, M. Passive Motor Learning: Oculomotor Adaptation in the Absence of Behavioral Errors. eNeuro 8, (2021).

37. Mendelsohn, A., Pine, A. \& Schiller, D. Between Thoughts and Actions: Motivationally Salient Cues Invigorate Mental Action in the Human Brain. Neuron 81, 207-217 (2014). 
bioRxiv preprint doi: https://doi.org/10.1101/2021.08.12.456140; this version posted August 13, 2021. The copyright holder for this preprint (which was not certified by peer review) is the author/funder, who has granted bioRxiv a license to display the preprint in perpetuity. It is made available under aCC-BY 4.0 International license.

Implicit adaptation of withheld movements - 26

626

627

628

629

630

631

632

633

634

635

636

637
38. Reddan, M. C., Wager, T. D. \& Schiller, D. Attenuating Neural Threat Expression with Imagination. Neuron 100, 994-1005.e4 (2018).

39. Herzfeld, D. J., Vaswani, P. A., Marko, M. K. \& Shadmehr, R. A memory of errors in sensorimotor learning. Science 345, 1349-1353 (2014).

40. Beers, R. J. van. Motor Learning Is Optimally Tuned to the Properties of Motor Noise. Neuron 63, 406-417 (2009).

41. Wu, H. G., Miyamoto, Y. R., Castro, L. N. G., Ölveczky, B. P. \& Smith, M. A. Temporal structure of motor variability is dynamically regulated and predicts motor learning ability. Nat Neurosci 17, $312-$ 321 (2014).

42. photonstorm.com, P. S.-. Phaser - Download - Phaser 3.24.1. https://phaser.io.

43. Tsay, J. S., Ivry, R. B., Lee, A. \& Avraham, G. Moving outside the lab: The viability of conducting sensorimotor learning studies online. NBDT 1-22 (2021) doi:10.51628/001c.26985. 


$$
\text { Implicit adaptation of withheld movements }-27
$$

\section{ONLINE METHODS}

Participants. Participants ( $n=213$, aged 18-35, 105 female) recruited from the research participation pools at Princeton/Yale University and Prolific provided informed consent, approved by each University's IRB. Ninety participants were excluded for failure to recall task instructions, as ascertained by a questionnaire at the end of the experiment, leaving 123 participants for our analysis. See the Questionnaire section below the Test phase sections for more details. We note that all the key results described here (significant learning after No-Movement trials) held with or without these exclusions. We note that the samples used for each experiment described in the text are around twice the size of similar studies in the literature, providing additional statistical power to compensate for the experiment being conducted remotely. ${ }^{39-41}$

Task Setup. Experiments were conducted remotely using a custom JavaScript web application based on Phaser3.24 (download available at ${ }^{42}$ ), similar to an approach previously described. ${ }^{43}$ Each participant viewed a single target located at either $45^{\circ}, 135^{\circ}, 225^{\circ}$, or $315^{\circ}$ (with target position counterbalanced across participants), 250 pixels from a central starting location. The target was visible throughout the experiment. Participants used an input device of their choice to control their computer cursor during center-out movements. One participant reported using a touchscreen device and was excluded from all analyses. The remaining participants reported using either a trackpad $(n=112)$, an optical mouse $(n=86)$, or a trackball $(n=14)$. A linear mixed model (LMM) did not show effects of Mouse Type on single-trial learning (STL), although we observed that participants using a trackpad exhibited longer reaction times than others, consistent with a previous report. ${ }^{43}$

Mouse position sampling rates depended on the exact hardware that each participant used to complete the task. Sampling rates were likely affected by features of the specific mouse used, along with features of the specific computer used, as computers may limit the rate at which the browser samples data in order to cope with limited processing power. In general, sampling rates were around $60 \mathrm{~Hz}$ (median \pm interquartile range across all 213 participants recruited: $62.46 \pm 2.17 \mathrm{~Hz}$ ), but ranged from $19.23 \mathrm{~Hz}$ to $249.69 \mathrm{~Hz}$. Note that the vast majority of sampling rates were near $60 \mathrm{~Hz}$ : Only $5 \%$ of mouse sampling rates were $<41.79 \mathrm{~Hz}$, and only $5 \%$ of mouse sampling rates were $>126.65 \mathrm{~Hz}$. 
Implicit adaptation of withheld movements -28

Baseline training phase. During a baseline phase, participants were instructed to move their mouse into a central starting location and then quickly make a straight, slicing movement through the target when it turned green. After a $100 \mathrm{~ms}$ delay following the completion of the movement, the cursor reappeared near the central starting location so that participants could return to that location to initiate the next trial.

670 Participants completed 5 of these trials with online and endpoint cursor feedback, followed by 5 trials without 671 visual feedback of the cursor location. Endpoint feedback was constituted by the cursor remaining at the position where it had passed the target radius for $50 \mathrm{~ms}$. Participants then completed 10 alternating trials on which the target turned green and stayed green (Execution, 'Go' trials) and on which the target turned

674 magenta $100 \mathrm{~ms}$ after turning green, signaling that participants should withhold their movement (NoMovement, 'No-Go' trials). After this baseline phase, participants were instructed to continue following these instructions for the remainder of the experiment. divided into 3-trial triplets (Fig. 1c). The first and last trials of all triplets were Go trials, and participants received neither online nor endpoint feedback about cursor location on these trials. The second trial of each triplet was either a Movement or a No-Movement trial. On Movement trials, participants either received rotated/error-clamped ${ }^{8}$ visual feedback $\left(15^{\circ}\right.$ clockwise [+] or counterclockwise [-], with sign randomized across trials) or veridical $/ 0^{\circ}$ error-clamped visual feedback of their cursor location. On No-Movement (NoGo) perturbation trials, participants viewed a brief animation of the cursor moving straight to the center of the target following a trajectory deflected by $15^{\circ}$ (clockwise or counterclockwise) from the target center.

686 Animation onset latency was set as a running median of the participant's reaction times on the previous 5 trials. Animation duration was set as a running median of the participant's movement times on the previous 5 trials. If a participant took longer than $400 \mathrm{~ms}$ to execute a movement, $800 \mathrm{~ms}$ to initiate the movement, 689 their reach trajectory changed by $>10^{\circ}$ during the movement, or the reach terminated $\geq 60^{\circ}$ away from the 690 target, they received a warning and a 4 s time-out. If a participant moved the cursor at all ( $>0$ pixels) on a 691 No-Movement trial, the trial was immediately aborted, and they received a warning and a 4 s time-out. The 692 No-Go manipulation was successful: Across the experiments, participants erroneously moved on $29.55 \pm$ 

planning movements on No-Go trials.

We used 6 possible triplet perturbation trial types (Movement/No-Movement: $\pm 15^{\circ}$ or $0^{\circ}$ ), each of which occurred 15 times throughout each session. Triplets were pseudorandomly presented within each block, with the constraints that a single rotation $\left( \pm 15^{\circ}\right.$ or $\left.0^{\circ}\right)$ could not occur on more than 2 consecutive triplets and that the same movement condition (i.e., Movement or No-Movement) could not occur on more than 3 consecutive triplets. Three repetitions of each triplet type occurred in blocks of 18 triplets, and participants received a break after each of these blocks.

Test phase: Rotation and Error-Clamp Experiments with $0^{\circ}$ Perturbations on Movement Trials (Fig. 3). Experiments were conducted as described above, with the exception of the details described in this section. For the experiments described in Fig. 3a-d, we used a reduced set of 3 possible triplet Perturbation trial types (No-Movement, $15^{\circ}$ clockwise error; No-Movement, $15^{\circ}$ counterclockwise error; Movement, $0^{\circ}$ in order to ensure that participants would reliably respond to the "Go" cue presented at the start of each trial. So, each No-Movement triplet type occurred 22 times, while the Movement triplet type occurred 44 times. Triplets were pseudorandomly presented within each block, with the constraints that a single nonzero rotation ( $15^{\circ}$ clockwise, $15^{\circ}$ counterclockwise) could not occur on more than 2 consecutive triplets.

For the experiments described in Fig. 3e-h, we used a set of 4 possible triplet Perturbation trial occurred 45 times.

717 Questionnaire: The questionnaire asked participants to attest whether or not 1) they changed how they moved the mouse after seeing the cursor miss the target, 2) their goal was to move the real mouse and not 
Implicit adaptation of withheld movements - 30

720

721

722

723

724

725

726

727

728

729

730

731

732

733

734

735

736

737

738

739

740

741

742

743

744

745

746

747

mouse) straight through the green targets. Participants could select the options, "True," "False," or "Not Sure."

Data analysis. Data were processed in Python 3.8.5. Trials with movement were excluded from analysis 1) if any of the reaches in the triplet were not straight (aspect ratio $>$ participant-wise mean +3 * participantwise standard deviation), 2) if the participant received any warning for failure to follow task instructions (see Feedback for failure to follow task instructions, above), or 3) if the triplet included a No-Movement No-Go perturbation trial with any detectable mouse movement ( $>0$ pixels).

Reach endpoint angle was computed as the angular distance between the center of the target and the point at which the mouse passed the target's radial distance. Because mouse sampling rates did not always allow us to measure mouse position at the exact target radius, we used the last sample before and the first sample after the mouse passed the target radius to compute an interpolated mouse position at the target radius, as described in a previous report. ${ }^{43}$ We note that analyses comparing these measures to measurements at the last sample of the reach (even when it was beyond the target) or the hand angle at peak velocity did not result in substantially different hand angle measurements or statistical outcomes.

Single-trial learning (STL) was measured as the difference between reach endpoint angle on the third and first trial of each triplet. For our initial analyses, the sign of STL corresponded to the direction of the relative change in hand angle, with clockwise changes in hand angle taking a positive sign and counterclockwise changes in hand angle taking a negative sign. When we collapsed STL data across rotation directions, we normalized the sign of STL so that changes in hand angle opposite the direction of the imposed rotation took a positive sign and changes in the direction of the rotation took a negative sign.

Statistics. Statistical tests were conducted in R (v. 4.0.3; packages rcompanion, rstatix, magrittr, ggplot2, ggeffects, MuMIn, ImerTest, Ime4, r2glmm, emmeans, stats). Data from 2 of the 4 experiments conducted did not satisfy assumptions of two-way repeated measures ANOVA (non-existence of extreme outliers, sphericity), so we employed a linear mixed modeling (LMM; R package ImerTest and Ime4) approach for our analyses. All LMM's included fixed effects of perturbation size and movement condition, as well as random effects of subject. Degrees of freedom were estimated using the Kenward-Rogers approach, and 

for the LMM factors (R package r2glmm). Post-hoc pairwise comparisons were performed between estimated marginal means computed from the LMM (R package emmeans). For these and all subsequent analyses, we corrected for multiple comparisons using the false-discovery rate approach to maintain experiment-wise alpha at 0.05 .

For one-off comparisons between samples or to distributions with 0-mean, we checked samples for normality. When samples were normally distributed, we ran t-tests and computed Cohen's d to report effect sizes for statistically significant results. Otherwise, we ran Wilcoxon-signed rank tests and measured effect sizes using the rank-biserial correlation.

Initial efforts to model the remembered STL and the effects of errors experienced on preceding trials on STL using LMM approaches suffered from singularity. Thus, we employed generalized linear models (GLM; R package stats) assuming underlying Gaussian distributions.

To investigate whether STL was remembered following Movement and No-Movement trials, GLMs with terms for the perturbation applied $\left(15^{\circ} \mathrm{CCW}, 0^{\circ}\right.$, or $\left.15^{\circ} \mathrm{CW}\right)$, movement condition (Movement or NoMovement), and their interaction were fit to triplet-wise remembered STL performance (hand angle on the first trial of triplet $t+1$ minus hand angle on the first trial of triplet $t$ ) for each participant. We then compared the distributions of coefficients for each term using Wilcoxon's signed-rank tests.

To investigate whether errors affected STL measurements beyond the triplet in which they occurred, GLMs with terms for errors on Movement trials and No-Movement trials in the current triplet or one triplet back, plus pairwise interactions for these 4 terms, were fit to triplet-wise STL performance for each participant. We then compared the distributions of coefficients for each term using Wilcoxon's signedrank tests. 